\title{
Portal Dosimetry as a patient specific Quality Assurance tool for Volumetric Arc Radiotherapy
}

\author{
Rathore V.. ${ }^{1 *}$, Mishra V. ${ }^{2}$, Choudhary V. ${ }^{3}$, Gautam G. ${ }^{4}$, Kushawaha P. ${ }^{5}$, Azad S. ${ }^{6}$, Kerketta M. ${ }^{7}$, \\ Chandrakar P. ${ }^{8}$, Singh R. ${ }^{9}$, Jain R. ${ }^{10}$ \\ DOI: https://doi.org/10.17511/ijmrr.2021.i01.04
}

1* V.B. Rathore, Associate Professor, $^{2}$ V.K Mishra, Assistant Professor, ${ }^{3}$ V Choudhary, Director-cum Professor and Dean, ${ }^{4}$ G.S. Gautam, Assistant Professor, ${ }^{5}$ P. Kushawaha, Registrar, ${ }^{6}$ S.K. Azad, Professor, ${ }^{7}$ M. Kerketta, Associate Professor, ${ }^{8}$ P.K. Chandrakar, Associate Professor, 9 R.S. Singh, Assistant Professor, 10 R.R. Jain, Assistant Professor; all authors are affiliated with the Department of Radiotherapy, Pt. J. N. M. Medical College and Regional Cancer Centre, Raipur, Chhattisgarh, India.

Introduction: Volumetric Arc Radiotherapy (VMAT) is an advanced technique. Calculations of VMAT plans are not so accurate even with State-of-Art dose calculation algorithms due to their complexity. Hence pre-treatment patient specific Quality Assurance (QA) of each VMAT plan is required. In the present study Electronic Portal Imaging Device (EPID) based portal dosimetry system was used for pre-treatment patient specific QA. Material and Methods: A total of 50 patients were chosen in this study. Verification plans of each patient were calculated for portal dosimetry then executed on the EPID system to measure the spatial distribution of radiation dose. Calculated and measured dose distribution were compared to evaluate Gamma Index (GI) passing criteria of Dose Difference (DD) of $3 \%$ and Distance-to-Agreement (DTA) of 3mm, Area Gamma $(\gamma \% \leq 1)>95 \%$, Average Gamma (gAve) $<0.5 \%$ and Maximum Gamma (gMax) $<3.5 \%$. Results: The mean values of Area Gamma ( $\%$. $\leq 1$ ) were observed to be varied from $99.14 \pm 0.23 \%$ to $99.87 \pm 0.18 \%$. The Mean Values of Average Gamma (gAve) are found to vary from $0.19 \pm 0.05 \%$ to $0.15 \pm 0.04 \%$ and the mean values of Maximum Gamma (gMax) found to be varied from $1.94 \pm 0.37 \%$ to $1.59 \pm 0.41 \%$. All the plans were passed the gamma index criteria with very good agreement. Thus the use of Portal Dosimetry for pre-treatment patient QA is found to be a very useful, quick, precise, efficient and effective pretreatment patient specific QA tool for VMAT treatment. Conclusion: Portal Dosimetry can be utilized for routine use for patient specific quality assurance for Volumetric Arc Radiotherapy treatment.

Keywords: Portal Dosimetry, Gamma Index, VMAT Quality Assurance, Patient-Specific Quality Assurance

\section{Corresponding Author}

V.B. Rathore, Associate Professor, Department of Radiotherapy, Pt. J. N. M. Medical College and Regional Cancer Centre, Raipur, Chhattisgarh, India. Email: rathorevb@rediffmail.com
How to Cite this Article

To Browse

Rathore VB, Mishra VK, Choudhary V, Gautam GS, Kushawaha P, Azad SK, Kerketta M, Chandrakar PK, Singh RS, Jain RR. Portal Dosimetry as a patient specific Quality Assurance tool for Volumetric Arc Radiotherapy. Int J Med Res Rev. 2021;9(1):29-33. Available From

https://ijmrr.medresearch.in/index.php/ijmrr/article/

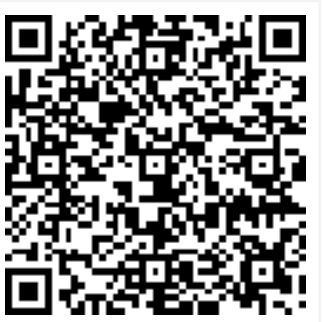
view/1238

$\begin{array}{cc}\begin{array}{c}\text { Manuscript Received } \\ 2020-12-24\end{array} & \begin{array}{c}\text { Review Round 1 } \\ 2021-01-04\end{array} \\ \text { Conflict of Interest } & \text { Funding } \\ \text { No } & \text { Nil }\end{array}$

Review Round 2 2021-01-14

Ethical Approval Yes
Review Round 3

Plagiarism X-checker $5 \%$
Accepted 2021-01-22

Note 


\section{Introduction}

Volumetric Arc Radiotherapy (VMAT) is an advanced radiotherapy technique that allows the prescribed radiation dose to precisely conform to the target volume while a minimum dose to the nearby organ at risk. VMAT modulated the photon beam by modulations of Multi-Leaf Collimator (MLC) leaf positions, Gantry rotation speed and dose rate simultaneously during rotations of gantry around the patient (1-2). This rotational therapy delivers prescribed dose in a relatively shorter duration and has better dose conformity, uniformity and normal organ sparing. (3-7)

Calculations of the small or irregular fields which are frequently used for VMAT are not accurate even with the state-of-the-art dose calculation algorithm (8), therefore patient specific Quality Assurance (QA) of every patient treated with VMAT techniques were performed before patient treatment (9-11). The delivery of the radiation beam to the tumour requires quality assurance for every plan before treatment of the patient using a $2 \mathrm{D}$ array or portal dosimetry (12). The most widely used form of pre treatment Quality Assurance for IMRT/VMAT generally consists of absolute dose measurement with ionization chamber combined with isodose distribution measurements in a phantom (13-15). Electronic Portal Imaging Device (EPID) offer advantages over other systems as it is attached to the gantry of the accelerator and hence reduced the duration of set up. In the present study EPID based portal dosimetry system was used as a pre treatment patient specific QA for VMAT plans treated at the Department of Radiotherapy, Regional Cancer Centre, Pt. J.N.M. Medical College, Raipur.

\section{Material and Methods}

A total of 50 patients were included in this study. The plan of each patient was with 2Arcs. These plans include treatment of carcinoma located at various sites. All selected plans were optimized and calculated using Eclipse Treatment Planning System (TPS) Ver 15.6.3 (Varian Medical Systems Palo Alto CA USA). Progressive Resolution Optimizer (PRO) algorithms were used to generate an optimal plan then the dose was calculated using Anisotropic Analytical Algorithm (AAA) with a $2.5 \mathrm{~mm}$ grid. This treatment was executed on Clinac DHX Linear Accelerator with millennium 120TM MLC (Multi-Leaf Collimator) and 6MV nominal Photon energy.
All plans were undergone pre-treatment Quality Assurance (QA) with Portal Dosimetry System (Varian Medical Systems Palo Alto CA USA) attached with Linear Accelerators (Clinac DHX). Portal Dosimetry System which is consists of a portal vision aSi1000 comprises an $8 \mathrm{~mm}$ thickness main plate, a thin copper slice $(1 \mathrm{~mm})$ and a $0.5 \mathrm{~mm}$ phosphor film. The detector panel has a pixel dimension and spatial resolution of $1024 \times 768$ and $0.392 \mathrm{~mm}$ per pixel respectively. Portal Dosimetry was commissioned and calibrated according to manufacturer protocol before using it for pretreatment patient specific QA.

The Verification plan of each patient was created for portal dosimetry using Portal Dose Image Prediction (PDIP) algorithms in Eclipse TPS with Source to Image Distance (SID) $100 \mathrm{~cm}$. Verification plans were then executed on the EPID system to measure the spatial distribution of radiation dose. Figure-1 shows dose distribution for a VMAT plan (2Arc) of a patient with carcinoma tongue.

Calculated and measured dose distributions were compared to evaluate its accuracy of delivery with pre-defined passing criteria of Gamma Index (GI). Figure-2 shows a graphical representation of predicted and measured dose with their superposition along with the collimator axis for the above-cited VMAT plan. The criteria of gamma evaluation (Dose Difference (DD) of $3 \%$ and Distance-To-Agreement (DTA) of $3 \mathrm{~mm}$ ) were set based on clinical experience.

Figure-1: Beam arrangements and dose distributions for VMAT plan of a patient with carcinoma tongue

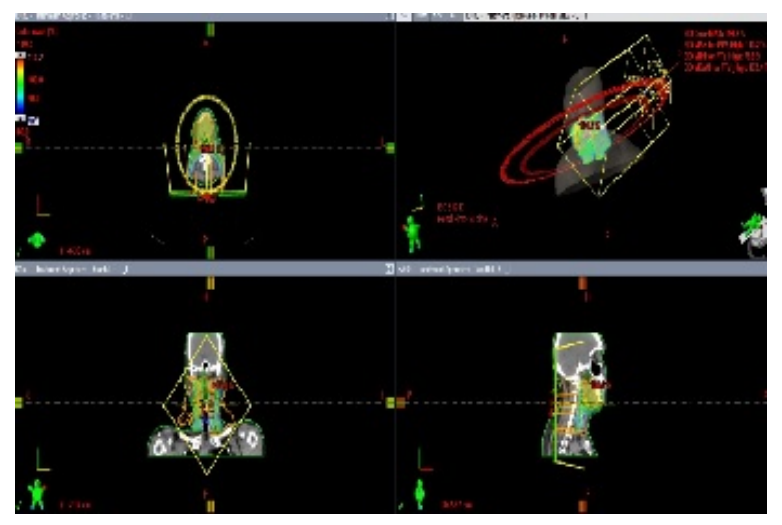

Figure-2 Graphical representation of predicted and measured dose with their superposition along collimator axis for the above-cited VMAT plan using Portal Dosimetry system. 


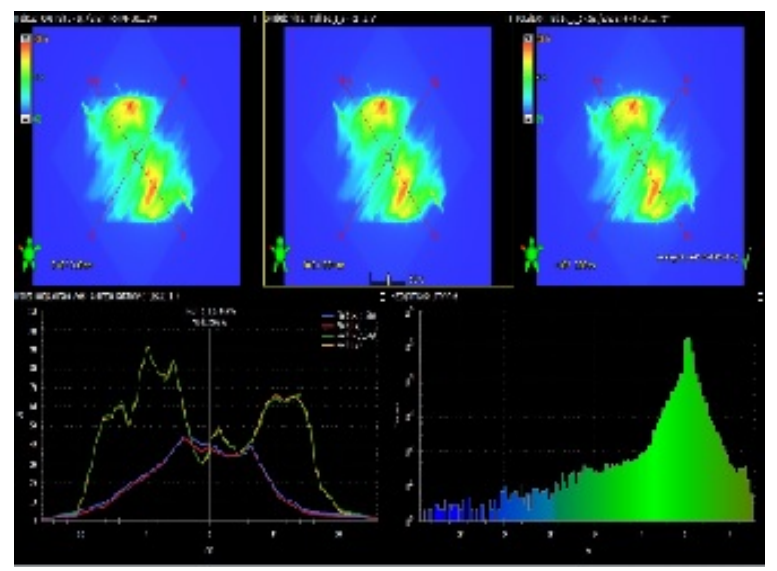

\section{Results}

The gamma index (GI) evaluation of the measured dose distributions in the Portal dosimetry/EPID system against the dose distribution predicted by the treatment planning system was performed. For each field of every plan, three gamma scaling parameters estimated are Area Gamma ( $\mathrm{g} \% \mathrm{~s}$ ), Average Gamma (gAve) and Maximum Gamma (gMax). Gamma index (Gamma criteria of Dose Difference (DD) 3\%, Distance to Agreement (DTA) $3 \mathrm{~mm}$ ) values of 50 patients with various treatment sites are tabulated in Table-1. The mean values of Area Gamma ( $\mathrm{g} \% \leq 1$ ) varies from $99.87 \pm 0.18 \%$ for Hard Palate to $99.14 \pm 1.20 \%$ for the VMAT plan of Bain tumours. The Mean Values of Average Gamma (gAve) are found to vary from $0.19 \pm 0.05 \%$ to $0.15 \pm 0.04 \%$ and the mean values of Maximum Gamma (gMax) found to be varied from $1.94 \pm 0.37 \%$ for a plan of carcinoma of the cervix to $1.59 \pm 0.41 \%$ for Brain tumours.

\section{Table-1: Data of Gamma parameters of portal dosimetry for VMAT plan with gamma passing criteria} DD 3\%/DTA 3mm

\begin{tabular}{|c|c|c|c|c|c|c|c|c|c|}
\hline \multirow[t]{2}{*}{ S. N } & \multirow[t]{2}{*}{$\begin{array}{c}\text { Site of } \\
\text { treatment }\end{array}$} & \multirow[t]{2}{*}{$\begin{array}{c}\text { Number of Arcs in each } \\
\text { plan }\end{array}$} & \multirow[t]{2}{*}{$\begin{array}{c}\text { Number of } \\
\text { patients }\end{array}$} & \multicolumn{2}{|c|}{ Area Gamma $(\mathrm{g} \% \leq 1)$} & \multicolumn{2}{|c|}{$\begin{array}{c}\text { Average Gamma } \\
\text { (gAve) }\end{array}$} & \multicolumn{2}{|c|}{ Maximum Gamma (gMax) } \\
\hline & & & & Mean & SD & Mean & SD & Mean & SD \\
\hline 1 & Hard palate & 2 & 5 & $\begin{array}{l}99.87(100.00 \\
-99.60)\end{array}$ & 8 & $0.15(0.20-0.10)$ & 0.04 & $1.64(2.30-1.13)$ & 0.55 \\
\hline 2 & Carcinoma Cervix & 2 & 6 & $99.45(99.90-98.10)$ & 0.6 & $0.18(0.23-0.14)$ & 0.03 & $1.94(2.48-1.42)$ & 0.37 \\
\hline 3 & Tongue & 2 & 13 & $99.75(100.00-98.70)$ & 0.3 & $0.19(0.26-0.12)$ & 0.04 & $1.90(3.42-0.95)$ & 0.65 \\
\hline 4 & Brain & 2 & 10 & $99.14(100.00-95.10)$ & 1.2 & $0.19(0.28-0.14)$ & 0.03 & $1.59(2.32-0.93)$ & 0.41 \\
\hline 5 & Buccal Mucosa & 2 & 11 & $99.73(100.00-98.70)$ & 0.3 & $0.18(0.29-0.10)$ & 0.04 & $1.75(2.80-1.02)$ & 0.50 \\
\hline 6 & Nasopharynx & 2 & 5 & $99.20(100.00-97.50)$ & 8.9 & $0.19(0.26-0.15)$ & 0.05 & $1.82(2.42-1.30)$ & 0.47 \\
\hline
\end{tabular}

In this study we have analyzed the data of EPID based Portal dosimetry for dose distribution verification as a pre-treatment patient specific quality assurance of VMAT plans to ensure acceptable accuracy of treatment delivery. The tolerance for passing criteria for VMAT plans was based on the per cent of pixel passing, Area Gamma $(\gamma \% \leq 1)>95 \%$, Average Gamma (gAve) $<0.5 \%$ and Maximum Gamma (gMax) $<3.5 \%$ with the passing criteria of Dose Difference (DD) 3\%, Distance to Agreement (DTA) $3 \mathrm{~mm}$.

In the present study we observed that all gamma parameters are within the tolerance limit which reveals that the comparisons of calculated and measured dose distributions are found within an acceptable level of accuracy for the delivery of a plan to treat the patient. If the gamma parameters go beyond the tolerance limit we need to determine the source of error and eliminate it to achieve maximum possible accuracy of plan and treatment delivery.

No considerable variation has been observed in the values of gamma parameters for various treatment sites covered in this study, which proves the consistency, reproducibility and suitability of portal Dosimetry system for patient specific quality assurance. Results of this study are found comparable to the values of gamma parameters reported by Ibrahim AG, et al (16) and Nainggolan $A$, et al (17) for dose distribution verifications of VMAT plans for various treatment sites using the Portal Dosimetry system. 


\section{Conclusions}

Portal dosimetry system for pre-treatment patientspecific QA overcomes the disadvantages of other dose distribution verification system like 2D array detector system which have a low resolution of detectors, and require more time to set up detectors, phantom and connect to the analyzing system.

The results of this study show very good agreement between TPS calculated dose distribution with measured on portal dosimetry system. Thus this study proves that portal dosimetry is a quick, precise, efficient and effective pre-treatment patient specific QA tool for VMAT treatment.

Portal Dosimetry can be utilized for routine use for patient specific quality assurance for Volumetric Arc Radiotherapy treatment.

\section{Author's contribution}

Dr V.B. Rathore: Primary investigator of this study, Data Analysis and manuscript preparation.

Mr. V.K Mishra: Helped in data collection, analysis and preparation of the manuscript.

Dr. V Choudhary: Guided to conduct this study and preparation of the manuscript

Mr. G.S. Gautam, Ms. P. Kushawaha, Dr. S.K. Azad, Dr. M. Kerketta, Dr P.K, Chandrakar, Dr R.S. Singh and Dr. R.R. Jain: Preparation of this manuscript.

All authors are critically reviewed and approved the final manuscript.

\section{Acknowledgements}

We would like to thanks Dr.Vishnu Dutt, Dean, Pt.J.N.M.Medical College, Raipur for his continuous encouragement and support for this study. We are also grateful to all the staff of the Department of Radiotherapy for their co-operation.

\section{Reference}

01. Brahme A. Optimisation of stationary and moving beam radiation therapy techniques. Radiother Oncol. 1988;12(2)129-40.

DOI: [Article] [Crossref]
02. Otto K. Volumatric modulated arc therapy- IMRT in a single gantry arc. Med Phys. 2008:35(1)310-7. DOI: [Article] [Crossref]

03. Cozzy L,Dinshaw KA, Shrivastava SK, et al. A treatment planning study comparing volumetric arc modulation with Rapid Arc and fixed field IMRT for cervix uteri radiotherapy. Radiotherapy Oncol. 2008;89(2)180-91.

DOI: [Article] [Crossref]

04. Fogliata A, Clivio A, Nicolini G, Venetti E and Cozzi L. Intensity modulation with photons for benign intracranial tumours- A planning comparision of volumetric single arc, helical arc and fixed gantry techniques. Radiother Oncol. 2008;89(3)254-62.

DOI: [Article] [Crossref]

05. Verbakel WF, Cuijpers JP, Hoffmans D, et al. Volumetric intensity-modulated arc therapy vs conventional IMRT in head-and-neck cancer- A comparative planning and dosimetric study. Int J Radia Oncol Biol Phys. 2009;74(1)252-259.

DOI: [Article] [Crossref]

06. Nicolini G, Clivio A, Fogliata A, et al. Simultaneous integrated boost radiotherapy for bilateral breast a treatment planning and dosimetric comparison for volumetric modulated arc and fixed field intensity modulated therapy. Radiol Oncol. 2009;4;27.

DOI: [Article] [Crossref]

07. Rao M, Yang W, Chen F, et al. Comparison of Elekta VMAT with helical tomotherapy and fixed field IMRT- Plan quality, Delivery efficiency and accuracy. Med Phys. 2010;37(3)1350-9.

DOI: [Article] [Crossref]

08. Park JM, Park SY, Kim H. Modulation index for VMAT considering both mechanical and dose calculation uncertainties. Phys Med Biol. 2015;60(18)7101-25.

DOI: [Article] [Crossref]

09. Fred A, Scherman JB, Fog LS, Munk of Rosenchold P. Patient QA systems for rotational radiation therapy- a comparative experimental study with intentional errors. Med Phys. 2013;40(3)031716.

DOI: [Article] [Crossref]

10. Heilmann G, Poppe B, Laub W. On the sentivity of common gamma-index evaluation methods to MLC misalignments in Rapidarc quality assurance. Med Phys. 2013;40(3)031702.

DOI: ‘Article] ‘Crossref] 
11. Kim JI, Park SY, Kim HJ, Kim JH, Ye SJ, Park JM. The sensitivity of gamma-index method to the positioning errors of high-definition MLC in patient-specific VMAT QA for SBRT. Radiat Oncol. 2014;9;167.

DOI: [Article] [Crossref]

12. Mehrb C, Chevillard C, Ksouri W, Fawzi M, Bollet M. Comparison between two different algorithms used for pretreatment QA via aSi Portal Images. J Appl Clin Med Phys. 2015;16;5202.

DOI: [Article] [Crossref]

13. Vieira SC, Dirkx ML, Heijmen BJ. De Boer HC SIFT- A method to verify the IMRT Fluence delivered during patient treatment using an electronic portal imaging device. Int J Radiation Oncology Biol Phys. 2004;60;981-248.

DOI: [Article] [Crossref]

14. Sharma DS, Mhatre V, Heigrujam M, Talapatra K, Mallik S. Portal Dosimetry for Pretreatment verification of IMRT Plan- A comparision with 2D ion chamber array. Journal of Applied Clinical Medical Physics. 2010;11;238-248.

DOI: [Article] [Crossref]
15. Low DA, Moran JM, Dempsey JF, Dong L, Oldam M. Dosimetry tools and techniques for IMRT, Med. Phys. 2011;38;1313-1326.

DOI: [Article] [Crossref]

16. Ibrahim AG, Mohammed IE,Zidan HM. Dosimetric comparision of Amorphous Silicon EPID and 2D Array detector for Pre-treatment Verification of Intensity Modulated Radiation Therapy. International journal of Medical Physics, clinical Engineering and Radiation Oncology. 2018;7;438-452.

DOI: [Article] [Crossref]

17. Nainggolan A, Pawiro SA. Dosimetric Evaluation of Volumetric Modulated Arc Therapy (VMAT) and intensity Modulated Radiotherapy (IMRT) using AAPM TG 119 Protocol. J Biomed Phys Eng. $2019 ; 9(4) 395-408$.

DOI: $10.31661 /$ jbpe.v0i0.839 [Crossref] 